\title{
Is Catheter Ablation Better Than Antiarrhythmic Drugs for the Treatment of Atrial Fibrillation?
}

\author{
Balaji Natarajan, MD ${ }^{1}$ Srishti Nayak, MD ${ }^{2}$ Ramdas G. Pai, MD ${ }^{1}$ \\ ${ }^{1}$ Department of Cardiology, University of California Riverside School \\ of Medicine, Riverside, California \\ 2 Department of Cardiovascular Medicine, Loma Linda University \\ School of Medicine, Loma Linda, California \\ Int J Angiol 2020;29:131-140.
}

Address for correspondence Ramdas G. Pai, MD, FACC, FRCP, Department of Cardiology, University of California Riverside School of Medicine, Riverside, CA 92507 (e-mail: ramdas.pai@medsch.ucr.edu).

Abstract
Keywords
- atrial fibrillation
- ablation
- radio frequency
- ablation
- stroke
- pulmonary vein
- left atrium
- survival

Long-standing atrial fibrillation is associated with significant morbidity including stroke and development of heart failure. Patients also report poor quality of life as a result of debilitating symptoms or treatment side effects from antiarrhythmic medications. Radio frequency or cryothermal mediated catheter ablation has a central role in the management of symptomatic patients with paroxysmal or persistent atrial fibrillation. Circumferential pulmonary vein isolation is vital to the success of this therapy and other ancillary techniques have been described, especially for persistent atrial fibrillation. Several randomized controlled studies have been reported over the last two decades studying important clinical outcomes in patients with atrial fibrillation. In this article, we aim to provide a review of the major studies that have helped define the role of catheter ablation in the management of symptomatic atrial fibrillation in patients with both diseased and structurally normal hearts.
Rhythm control of atrial fibrillation (AF) is usually preferred in symptomatic patients and can be achieved either by using antiarrhythmic drugs (AADs) or by catheter ablation (CA) using either radio frequency or cryothermal energy. Vaughan Williams Class IC and Class III AAD are most commonly used, and they have significant side-effect profile including risk of ventricular arrhythmias, major contraindications, drug-drug interactions, and systemic toxicity, apart from poor overall efficacy. Over the past decade, circumferential pulmonary vein isolation has evolved from being a "novel" concept into a routine strategy to help manage patients with symptomatic AF. The primary aim of CA is to electrically isolate ectopic premature impulses that serve to trigger AF and eliminate them at the site of their origin around the pulmonary veins. Additional techniques to modify the left atrial substrate such as linear ablations of the left atrial posterior wall, focal complex fractionated atrial electrogram-guided ablations, ablation of ganglionic plexi, and areas of scar and other nonpulmonary vein triggers (superior vena cava or interatrial septum) have shown additive benefit, albeit at the risk of greater procedure-related complications. The purpose of this article is to provide a concise review of the compelling literature that has helped shape current guidelines for the use of CA in the management of AF, and its role in special populations.

The first-time success rate of CA in atrial flutter is excellent and ranges in the 90 to $95 \%$. In contrast, this is 50 to $70 \%$ in AF and improves to 80 to $90 \%$ with repeat procedures. Success of therapy is significantly much better in patients with paroxysmal AF compared with those with persistent AF. This largely explains the 2014 American Heart Association/ American College of Cardiology/Heart Rhythm Society (AHA/ ACC/HRS) recommendations for management of AF. CA has a Class I indication in symptomatic AF patients who are refractory or intolerant to at least 1 Class I/III AAD if paroxysmal, Class IIa if persistent and Class IIb if of the long-standing persistent ( $>12$ months) type. Additionally, CA may also be attempted as the first rhythm control strategy prior to trial of AAD in case of recurrent symptomatic paroxysmal (Class IIa) or persistent AF (Class IIb), although 


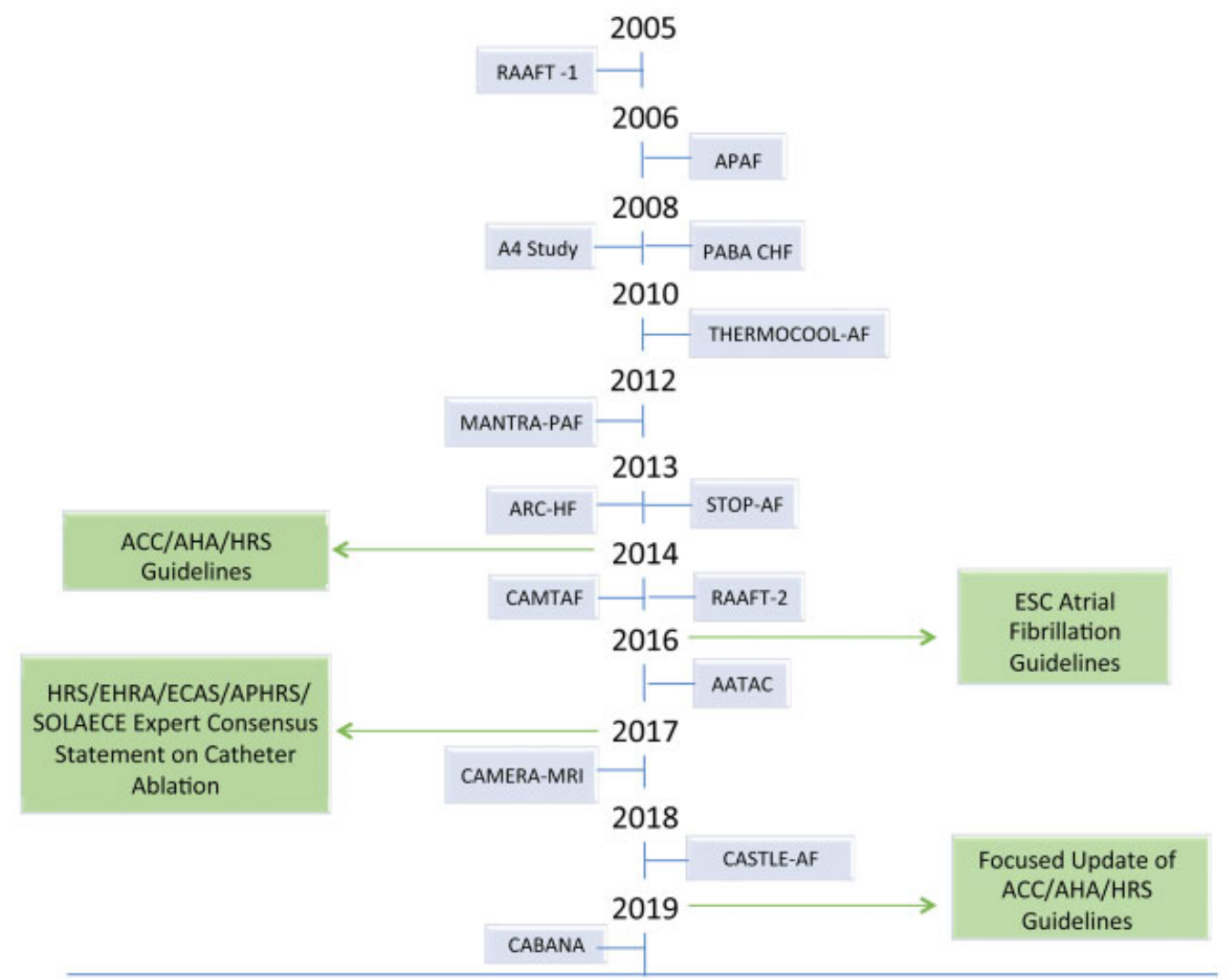

Fig. 1 Timeline of major randomized controlled trials characterizing the role of catheter ablation in symptomatic patients with atrial fibrillation.

we do not have strong evidence for this recommendation. ${ }^{1}$ The 2016 ESC $^{2}$ and 2017 HRS/EHRA/ECAS/APHRS/SOLAECE ${ }^{3}$ expert consensus statement on catheter and surgical ablation of AF also endorse the above recommendations. We will discuss the major randomized controlled trials (RCTs) that shaped the above guidelines in the following sections (-Fig. 1). Apart from chronicity and pattern of AF, patient characteristics such as advanced age, presence of structural heart disease such as heart failure (HF) and dilated left atrium (LA), presence of other comorbidities such as sleep apnea and obesity, and more importantly, operator volumes and experience also dictate success with these procedures. The role of CA in various patient populations is summarized (-Table 1).

\section{Role of Catheter Ablation in Structurally Normal Hearts}

Several small RCTs of symptomatic patients who have previously failed or intolerant to AAD have shown that CA is associated with less incidence of early and late AF recurrence, ${ }^{4-7}$ reduction in AF duration ${ }^{6,7}$ and frequency of episodes, ${ }^{4}$ decreased need for hospitalizations, ${ }^{6,8}$ and have shown overall improvement in quality of life (QOL) and functional capacity measured through various standardized metrics. $^{4,7}$ Data reported from a large Canadian Registry showed that more than $50 \%$ patients with paroxysmal AF will eventually progress to persistent AF or die from an event within 10 years. $^{9}$ Also, due to progressive left atrial remod- eling and fibrosis, success of maintaining sinus rhythm declines with longer duration of disease (AF begets AF). This raises an important question; should we ablate patients early in the course of their disease? The RAAFT- 1 study ${ }^{10}$ sought to evaluate a strategy of initial CA compared with AAD therapy in a group of 70 treatment naïve patients with symptomatic AF. Although small, this study reported lower AF burden, reduced cardiovascular hospitalization, and improved QOL in AF patients treated with an initial strategy of CA at 1-year follow-up. A much larger MANTRA-PAF study ${ }^{11}$ $(n=294)$ found that early CA was associated with greater freedom from $A F$ recurrence and symptomatic recurrence at 2 years, and this effect was sustained even at 5-year followup in patients with paroxysmal AF. The RAAFT- 2 study ${ }^{12}$ also reported similar findings at 2-year follow-up but observed much higher complication rates with early CA (overall rate of $9 \%$ with a $6 \%$ incidence of cardiac tamponade) when compared with contemporary studies. However, these studies were hampered by slow recruitment due to highly selected study populations, routine protocol deviations by the subjects, differences in measured primary outcomes, and wide differences in CA techniques and operator experience. More importantly, most RCTs were underpowered to report hard outcomes such as mortality and stroke rates. This has led to confusion among referring providers and overall a lower acceptance of CA as an initial strategy in symptomatic AF patients among electrophysicians.

In contrast, the data are far more compelling in favor of CA for patients who have failed or unable to tolerate prior 


\begin{tabular}{|c|c|c|c|c|c|c|c|c|c|}
\hline 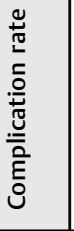 & 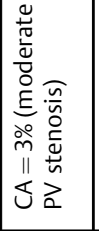 & 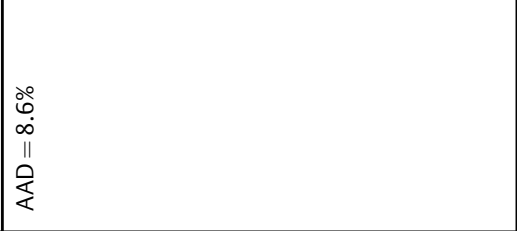 & \multicolumn{3}{|c|}{ 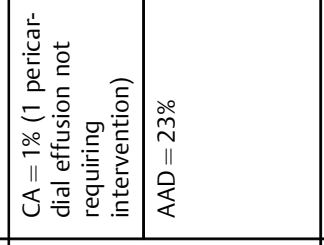 } & \multicolumn{2}{|c|}{ 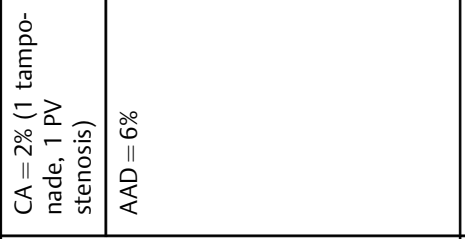 } & \multicolumn{2}{|c|}{ 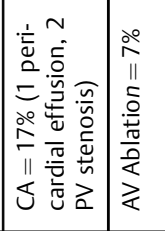 } \\
\hline 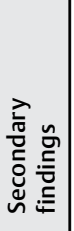 & \multicolumn{2}{|c|}{ 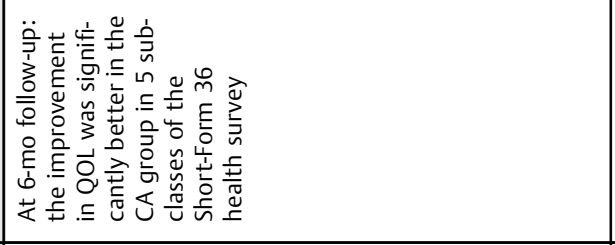 } & \multicolumn{3}{|c|}{ 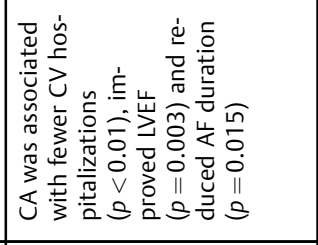 } & \multicolumn{2}{|c|}{ 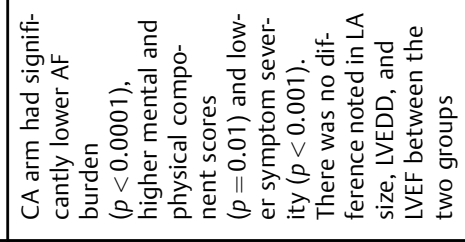 } & \multicolumn{2}{|c|}{ 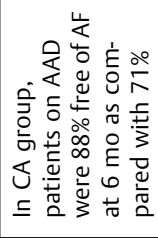 } \\
\hline 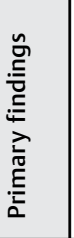 & \multicolumn{2}{|c|}{ 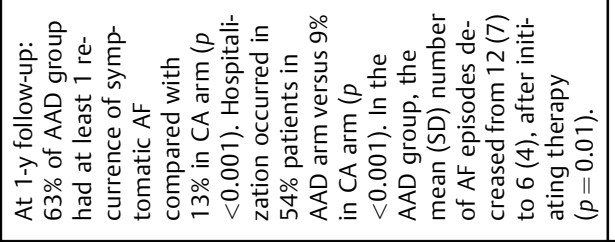 } & \multicolumn{3}{|c|}{ 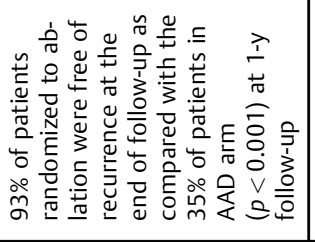 } & \multicolumn{2}{|c|}{ 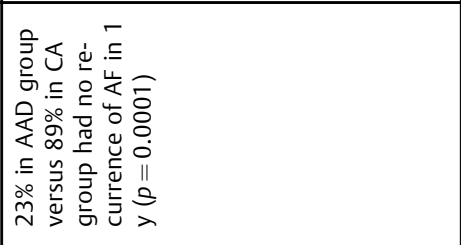 } & \multicolumn{2}{|c|}{ 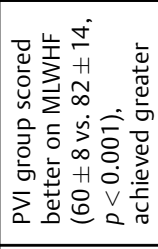 } \\
\hline 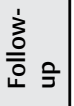 & \multicolumn{2}{|c|}{$\stackrel{\stackrel{O}{E}}{\simeq}$} & \multicolumn{3}{|l|}{$\begin{array}{l}\stackrel{O}{E} \\
\stackrel{\simeq}{\simeq} \\
\end{array}$} & \multicolumn{2}{|l|}{$\begin{array}{l}\stackrel{̊}{E} \\
\stackrel{\text { I }}{\simeq}\end{array}$} & \multicolumn{2}{|c|}{$\begin{array}{l}\stackrel{\circ}{E} \\
\text { D }\end{array}$} \\
\hline 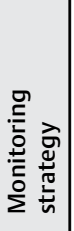 & \multicolumn{2}{|l|}{ 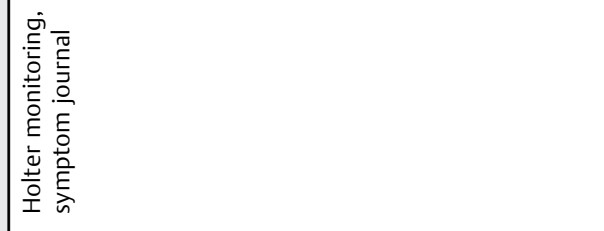 } & \multicolumn{3}{|c|}{ 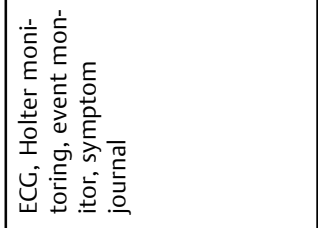 } & \multicolumn{2}{|c|}{ 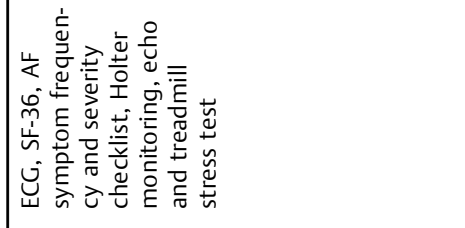 } & \multicolumn{2}{|c|}{ 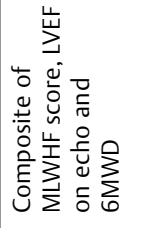 } \\
\hline 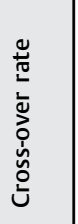 & \multicolumn{2}{|c|}{\begin{tabular}{|l} 
\\
\\
$\check{0}$ \\
$\check{0}$ \\
2
\end{tabular}} & \multicolumn{3}{|c|}{ 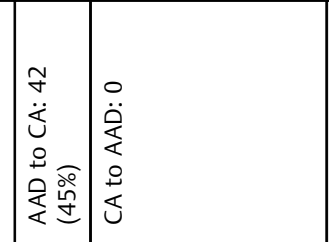 } & \multicolumn{2}{|l|}{ 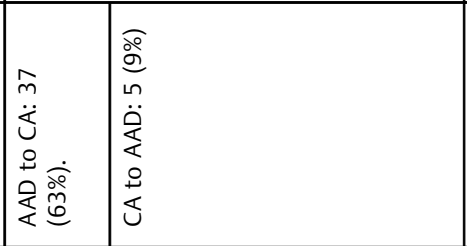 } & \multicolumn{2}{|l|}{ 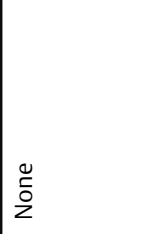 } \\
\hline 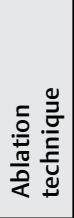 & \multicolumn{2}{|c|}{$z_{2}$} & \multicolumn{3}{|l|}{ ¿ } & \multicolumn{2}{|l|}{ ¿ } & \multicolumn{2}{|c|}{ 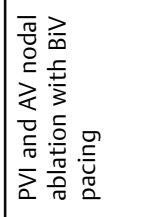 } \\
\hline 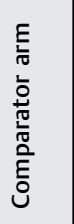 & \multicolumn{2}{|l|}{ 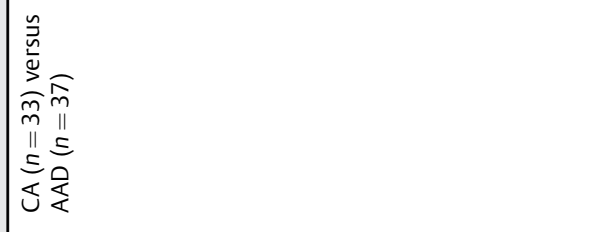 } & \multicolumn{3}{|c|}{ 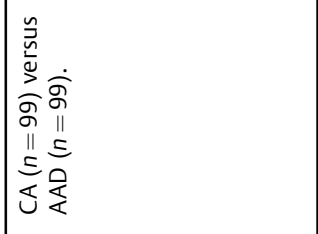 } & \multicolumn{2}{|c|}{ 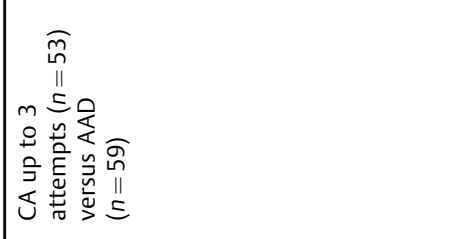 } & \multicolumn{2}{|c|}{ 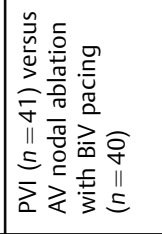 } \\
\hline 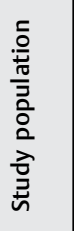 & \multicolumn{2}{|c|}{ 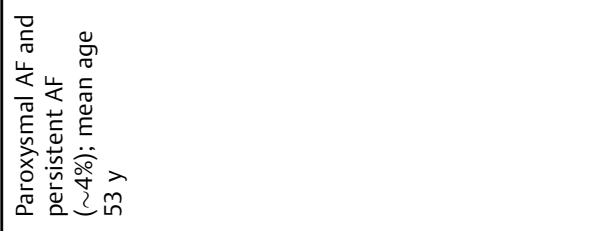 } & \multicolumn{3}{|c|}{ 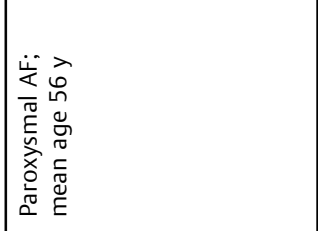 } & \multicolumn{2}{|l|}{ 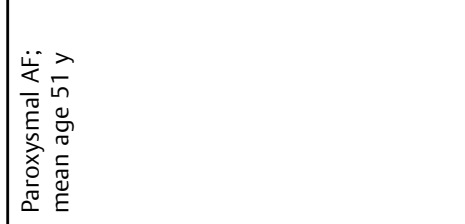 } & \multicolumn{2}{|c|}{ 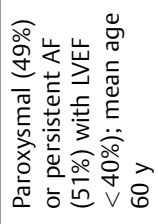 } \\
\hline$=$ & \multicolumn{2}{|l|}{ 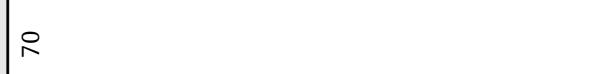 } & \multicolumn{3}{|l|}{ 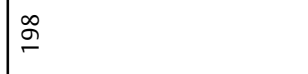 } & \multicolumn{2}{|l|}{$\stackrel{\cong}{\cong}$} & \multicolumn{2}{|l|}{$\bar{\infty}$} \\
\hline गे & 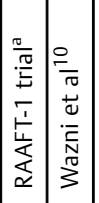 & 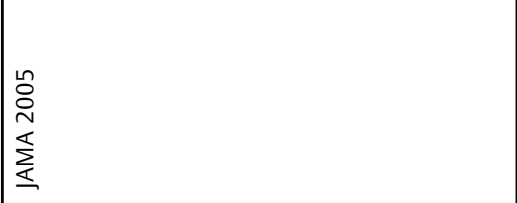 & 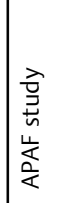 & 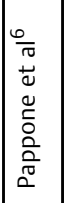 & 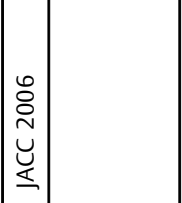 & 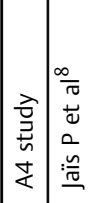 & 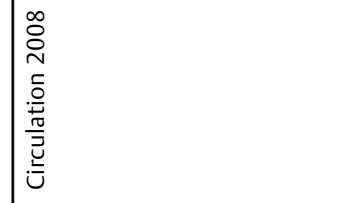 & 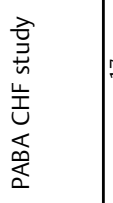 & 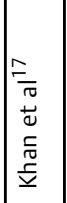 \\
\hline
\end{tabular}


134 AF Ablation Natarajan et al.

\begin{tabular}{|c|c|c|c|c|c|c|c|c|c|c|}
\hline 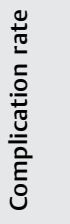 & & \multicolumn{2}{|c|}{ 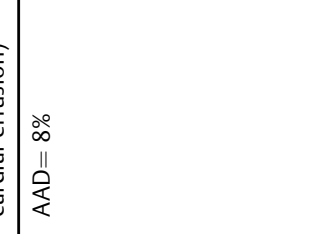 } & \multicolumn{2}{|c|}{ 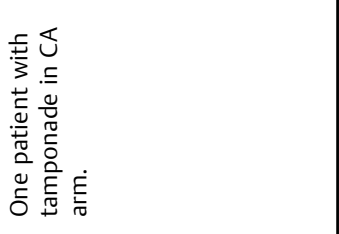 } & 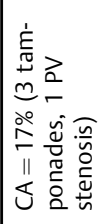 & \multicolumn{2}{|l|}{ 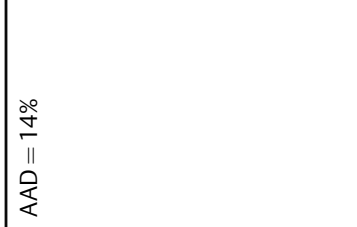 } & \multicolumn{2}{|c|}{ 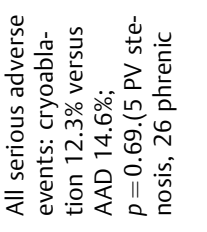 } \\
\hline 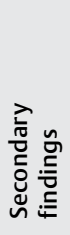 & 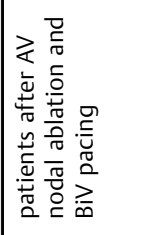 & \multicolumn{2}{|c|}{ 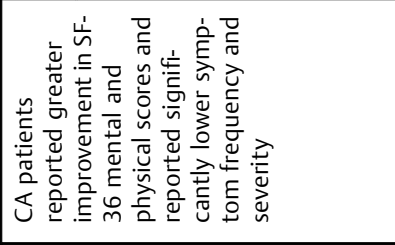 } & \multicolumn{2}{|c|}{ 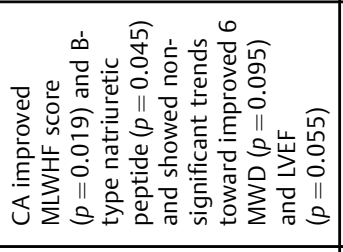 } & \multicolumn{3}{|c|}{ 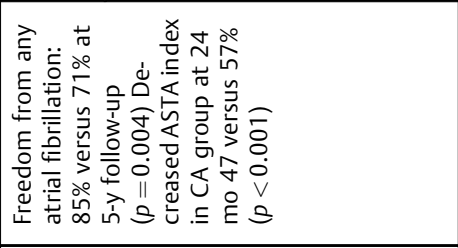 } & \multicolumn{2}{|l|}{$\begin{array}{l}0 \\
\stackrel{0}{0} \\
2\end{array}$} \\
\hline 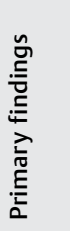 & 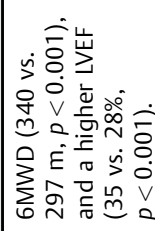 & \multicolumn{2}{|c|}{ 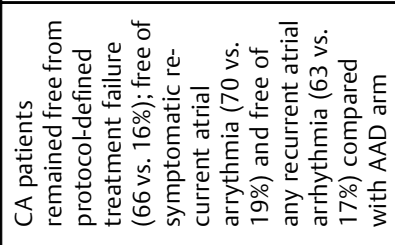 } & \multicolumn{2}{|c|}{ 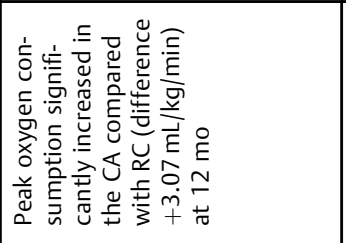 } & \multicolumn{3}{|c|}{ 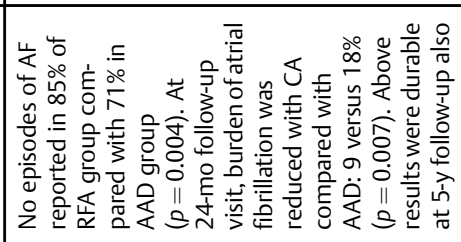 } & \multicolumn{2}{|c|}{ 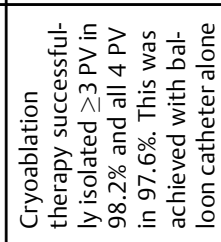 } \\
\hline $\begin{array}{l}\text { 定 } \\
\overline{\overline{0}}\end{array}$ & & \multicolumn{2}{|l|}{$\begin{array}{l}\circ \\
\text { Ĕ } \\
\sigma\end{array}$} & \multicolumn{2}{|c|}{$\stackrel{\stackrel{O}{E}}{\underset{\simeq}{\simeq}}$} & \multicolumn{3}{|l|}{$\begin{array}{l}\stackrel{\circ}{E} \\
\stackrel{+}{\sim}\end{array}$} & \multicolumn{2}{|l|}{ 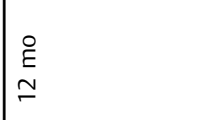 } \\
\hline 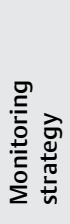 & & \multicolumn{2}{|c|}{ 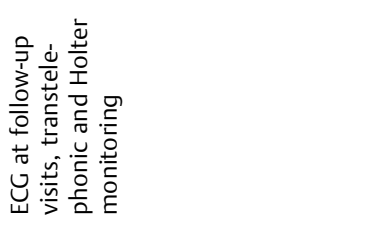 } & \multicolumn{2}{|c|}{ 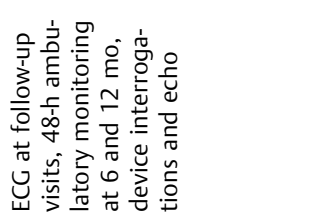 } & \multicolumn{3}{|c|}{ 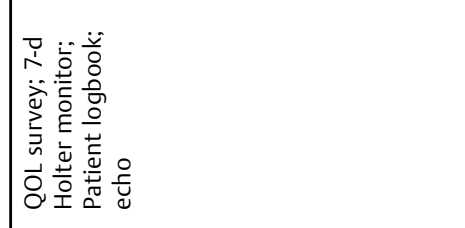 } & \multicolumn{2}{|c|}{ 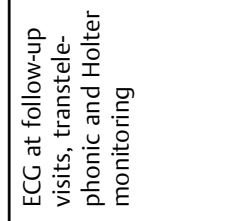 } \\
\hline 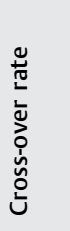 & & \multicolumn{2}{|c|}{ 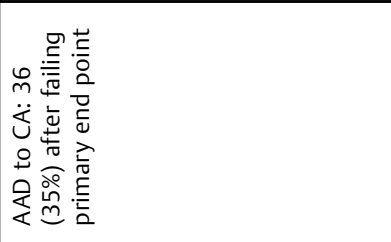 } & & 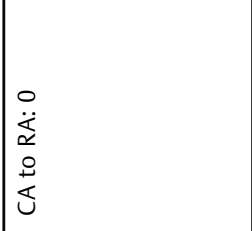 & 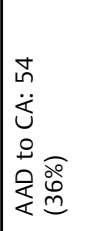 & 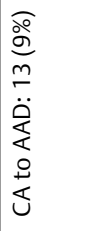 & & \multicolumn{2}{|c|}{ 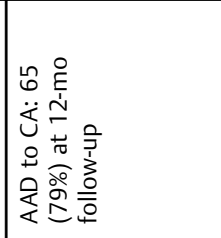 } \\
\hline 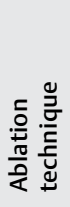 & & \multicolumn{2}{|l|}{ 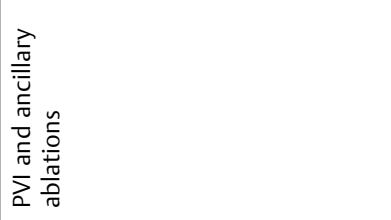 } & \multicolumn{2}{|l|}{ 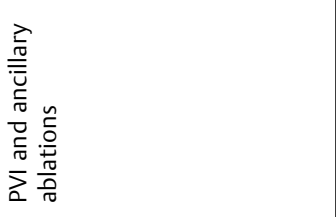 } & \multicolumn{3}{|l|}{ 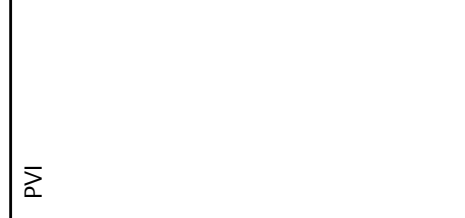 } & \multicolumn{2}{|l|}{ 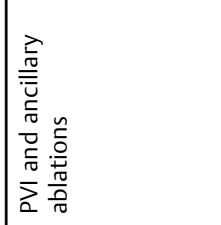 } \\
\hline 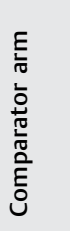 & & \multicolumn{2}{|l|}{ 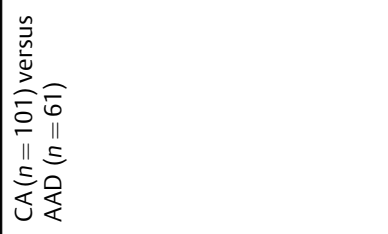 } & \multicolumn{2}{|c|}{ 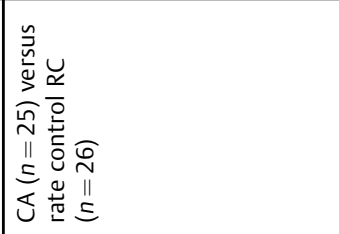 } & \multicolumn{3}{|c|}{ 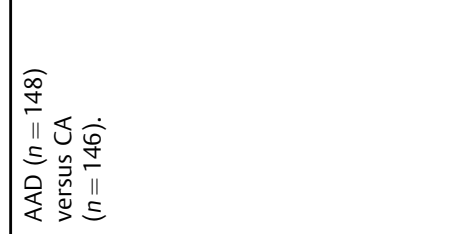 } & \multicolumn{2}{|c|}{ 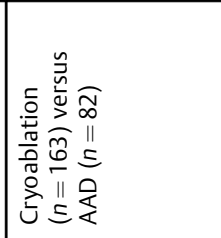 } \\
\hline 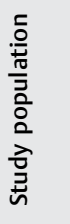 & & \multicolumn{2}{|l|}{ 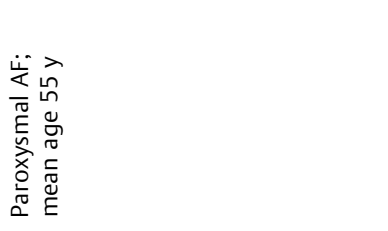 } & \multicolumn{2}{|c|}{ 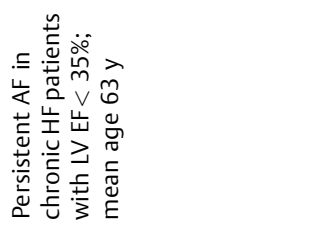 } & \multicolumn{3}{|l|}{ 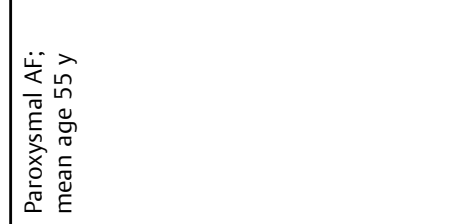 } & \multicolumn{2}{|c|}{ 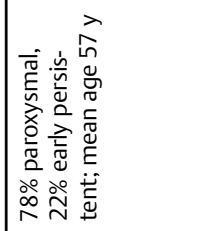 } \\
\hline$=$ & & \multicolumn{2}{|l|}{$\underline{6}$} & \multicolumn{2}{|l|}{ in } & \multicolumn{3}{|l|}{ 芯 } & \multicolumn{2}{|l|}{$\stackrel{\stackrel{\sim}{\sim}}{\sim}$} \\
\hline 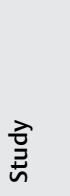 & 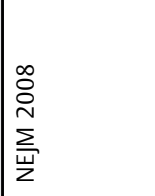 & 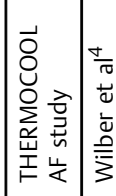 & 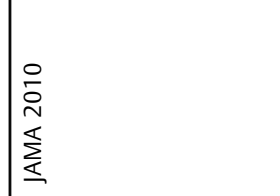 & 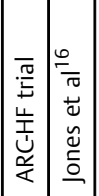 & $\begin{array}{l}m \\
\stackrel{n}{\tilde{N}} \\
\stackrel{u}{\underline{u}} \\
\underline{\underline{u}}\end{array}$ & 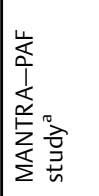 & 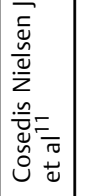 & 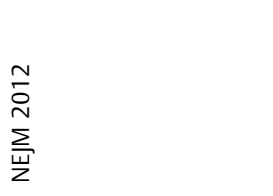 & 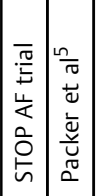 & 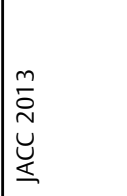 \\
\hline
\end{tabular}




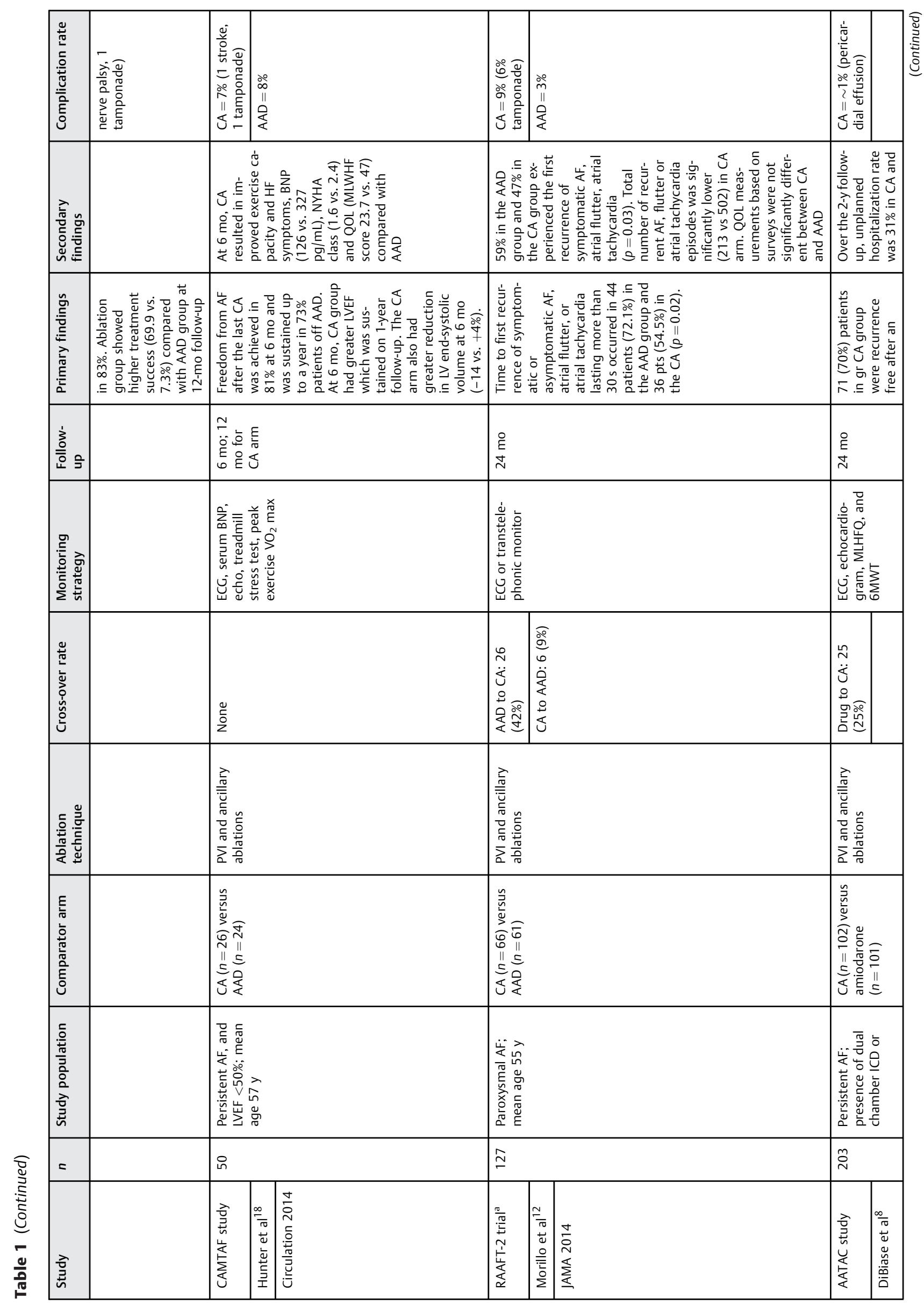




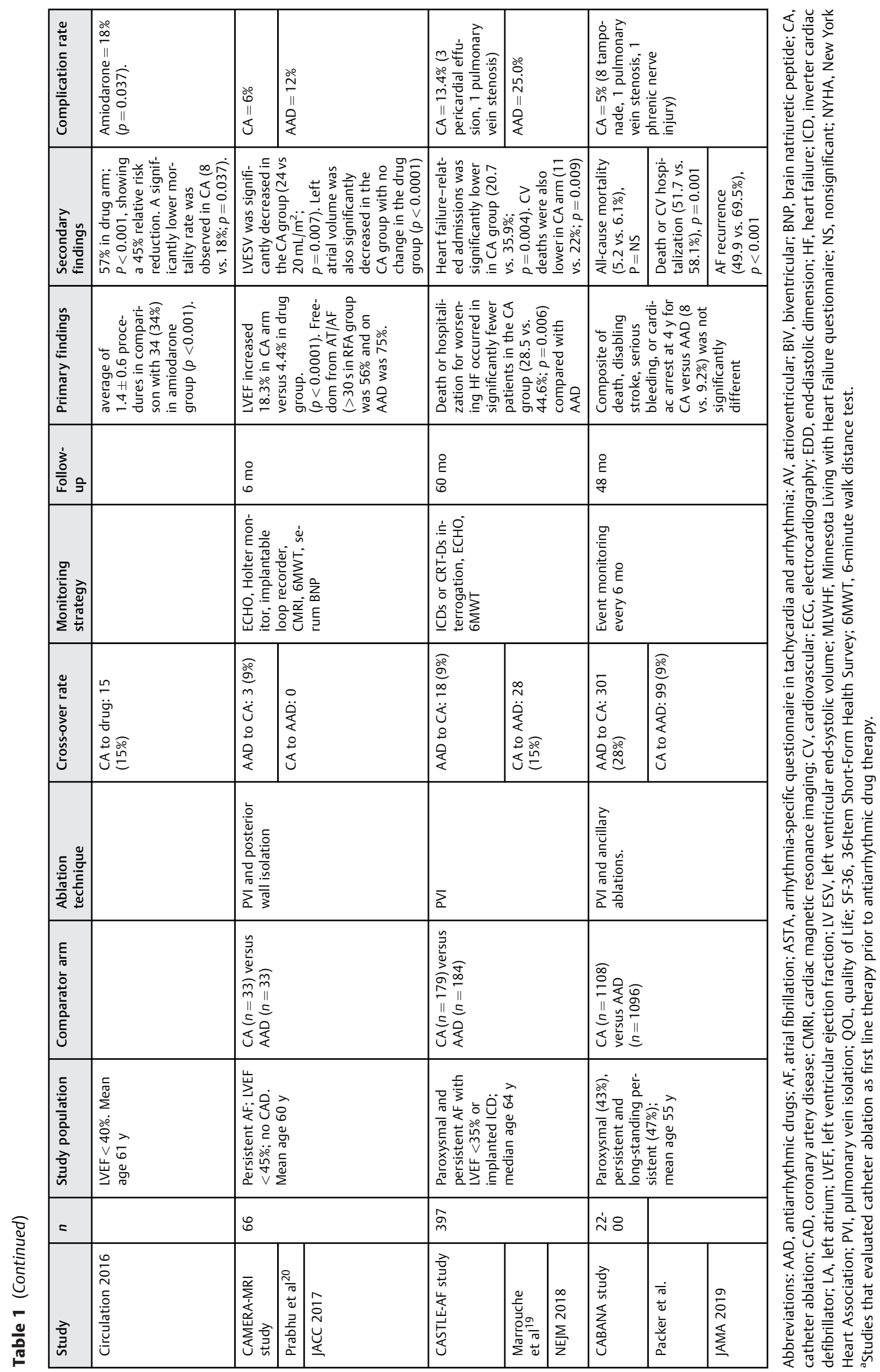


antiarrhythmic therapy. The much-anticipated CABANA trial was reported last year, and for the first time, a study provided long-term data on hard end points (death, stroke, and cardiac arrest) for ablation versus drug therapy in a large group of patients $(n=2,204)$ with paroxysmal or persistent AF who had previously failed AAD therapy. The primary outcome (composite of death, disabling stroke, serious bleeding, or cardiac arrest) was not significantly different between the groups ( $8 \%$ in CA vs. $9 \%$ in AAD group; $p=0.30$ ). None of the isolated primary end points achieved statistical significance either; however, the investigators noted a significant reduction in AF burden and recurrence, as well as reduction in hospitalizations and improvement in QOL in patients after CA at 5 years. Also, this study documented a significantly lower incidence of major complications $(\sim 5 \%)^{13}$ such as cardiac tamponade, pulmonary vein stenosis, and phrenic nerve injury in the CA ablation group as compared with previous RCTs. This supports the notion that CA, if done in high volume centers by experienced providers, can be a relatively safe procedure and helps to improve symptoms and QOL. Considering that there were large protocol deviations among the study participants (study crossover rate: ablation to drug-9.2\%; drug to ablation-27.5\%), the CABANA trial authors had also reported on "as-treated" analysis, where the groups were analyzed based on the treatment received instead of their originally assigned group. In contrast to the intention-to-treat analysis, the results overwhelmingly favored ablation for the composite as well as individual components of the primary end point. This became a point of controversy among experts in the field and the jury was divided regarding the mortality benefit of CA. Noseworthy et $\mathrm{al}^{14}$ reported their findings from a retrospective cohort analysis of a large national database by replicating the CABANA study protocol and included patients who underwent CA within the CABANA trial recruitment period (2009-2016). In this large group of patients $(n=183760)$, the primary outcome (composite of death, disabling stroke, serious bleeding or cardiac arrest) strongly favored CA compared with AAD (hazard ratio [HR]: $0.75,95 \%$ confidence interval (CI): $0.70-0.81 ; p<0.001]$. Individual primary end points were also significantly lower in CA arm except for major bleeding. This data, although supports the findings from the "as-treated" analysis of the CABANA trial, needs to be interpreted with caution due to their observational nature and the chance for residual confounding. Nevertheless, this study supports the argument that CA might have a mortality benefit in the "real world" and the findings of the "as-treated" analysis from CABANA cannot be entirely discounted as a chance occurrence.

\section{Role of Catheter Ablation in Patients with Heart Failure}

AF and HF co-exist in many patients and episodes of rapid AF in patients with pre-existing HF can worsen cardiac output due to loss of "atrial kick" and further impaired diastolic filling. Conversely, long-standing HF can cause increases in filling pressures and LA stretch from volume overload, disrupt ionic currents that regulate intracellular calcium, and cause imbalances in autonomic function that can facilitate the genesis of AF. Moreover, HF has also been shown to cause interstitial fibrosis and remodeling of the LA, and this can serve as a substrate to propagate AF thereby setting up a vicious cycle of worsening HF. ${ }^{15}$ AADs have a limited role to play in HF due to potential toxicities, and this led to interest in the potential role of CA to restore sinus rhythm in these patients. Early studies have shown us that CA can cause improvement in the left ventricular ejection fraction (LVEF) ( $\sim 5-10 \%$ ) from baseline, decrease brain natriuretic peptide levels and HF hospitalizations and improve both QOL and functional capacity in the form of increase in peak oxygen consumption and 6-minute walk distances, an important prognostic indicator. ${ }^{16-19}$ However, most studies were underpowered to study mortality. A good example is the AATAC study ${ }^{8}$ that found a $56 \%$ relative risk reduction for mortality with CA when compared with amiodarone therapy at 2-year follow-up, but it was underpowered to study this end point $(n=203)$. Additionally, the PABA CHF ${ }^{17}$ study showed superiority of CA over atrioventricular (AV) nodal ablation with biventricular pacing in terms of freedom from AFat 6 months, and improvements in QOL, functional capacity, and LVEF from baseline in patients with LVEF $<40 \%$.

The CASTLE-AF trial ${ }^{19}$ was the first adequately powered RCT to report a mortality benefit with CA in HF patients. This study enrolled 397 patients with symptomatic paroxysmal or persistent AF and HF with a LVEF of $\leq 35 \%$. The composite primary end point of all-cause mortality and unplanned HF hospitalization was significantly lower in CA arm versus medical therapy-rate or rhythm control (28.5 vs. $44.6 \%$; $p=0.007)$ at a median follow-up of 37.8 months. Additionally, the CA ablation group also showed a median LVEF improvement of $8 \%(2.2-19.1 \%)$ and greater freedom from AF (63.1 vs. $21.7 \% ; p<0.001)$ at 5-year follow-up visit. Another RCT presented around the same time; the CAMERA-MRI study ${ }^{20}$ challenged the notion that rate control is equivalent to rhythm control in persistent AF patients with LV dysfunction. This study confirmed improvement in LVEF and favorable chamber remodeling with CA, but additionally also observed that ablated patients with myocardial scar (presence of delayed gadolinium enhancement on cardiac magnetic resonance imaging) had greater improvements in absolute LVEF by $10.7 \%$ and normalization of LV function at 6 months ( 73 vs. $29 \% ; p=0.0093$ ). Interestingly, although the CABANA trial was a negative study overall, a recent abstract presented by the authors at the HRS meeting described the outcomes in $886 \mathrm{HF}$ patients (40\% of the study population). CA showed a significant reduction in the primary end point (HR: 0.66; 95\% CI: 0.43-0.99) and in all-cause mortality (HR: 0.59; 95\% CI: 0.36-0.96). Additionally, AF recurrence was also much improved after CA (HR: $0.58 ; 95 \% \mathrm{CI}: 0.44,0.75) .{ }^{21}$ The compelling data from above-discussed RCTs have secured a Class Ila recommendation in the 2016 ESC guidelines ${ }^{2}$ and a Class IIb recommendation in the 2019 focused update of the AHA/ACC/HRS guideline for the management of patients with $\mathrm{AF}$ and $\mathrm{HF}$ with reduced $\mathrm{EF}$ (HFrEF). ${ }^{22}$ Although the prevalence of $A F$ is the same irrespective of whether $E F$ is 
reduced or preserved, we do not have sufficient data on the efficacy of CA in HF with preserved $\mathrm{EF}$ (HFpEF) population at this time. However, a recent analysis of retrospective registry data from the Mayo AF Symptom Inventory ${ }^{23}$ showed that there were no significant differences in AF burden or functional improvement between HFrEF and HFpEF patients, suggesting that this heterogenous group of patients might also benefit from CA.

\section{Role of Catheter Ablation in Special Populations}

Certain high-risk populations are under-represented in trials, and hence adequate evidence is not available to guide recommendations for $\mathrm{CA}$ in these special groups.

1. AF in patients at extremes of age: The elderly ( $>80$ years of age) patients have a high prevalence of AF and usually have multiple associated comorbidities that drive the severity of their symptoms. Especially, they have a high risk of stroke and data strongly support anticoagulation in this population as reflected in the CHA2DS2-VASc scoring system (where age $>75$ years is considered a major risk factor). Multiple studies have reported similar outcomes with CA in the elderly when compared with the younger population, and the guidelines do not support a less aggressive strategy based on age. However, these patients found suitable for CA should be carefully selected keeping in mind that there is higher risk of post-procedure complications and higher need for AAD postablation. In contrast, young patients (age $<45$ years) have lower rates of AF recurrence and lower procedural complication rates, possibly due to lesser comorbid conditions when compared with the older AF population undergoing CA. Based on unpublished subgroup analysis from the CABANA trial with patients stratified as $<65,65$ to 74 and $>75$ years of age, it was reported that recurrent $A F$ was reduced equally in all three groups, but the younger population $(<65$ years) showed significant reduction in all-cause mortality (HR: 0.41 ; 95\% CI: 0.19-0.90). CA could be a potential firstline rhythm control option for young patients, but this is not supported by strong evidence at this time.

2. AF in hypertrophic cardiomyopathy (HCM): Development of AF in HCM patients is more common in the general population and is associated with high mortality. Moreover, AF episodes are poorly tolerated and there is a high stroke risk in HCM patients. A rate control strategy is usually preferred first, with the addition of AAD such as amiodarone or disopyramide for rhythm control. Small studies have shown that CA in HCM patients is effective to suppress AF recurrences, especially in patients with paroxysmal AF and small atria, but need for repeat procedures is high. CA currently has a Class IIa recommendation from all professional society guidelines in HCM patients who fail or do not tolerate initial AAD therapy.

3. AFin the presence of an accessory pathway: Incidence of AF is $\sim 15 \%$ over 10 years in patients with Wolff-ParkinsonWhite syndrome and there is high risk of degenerating into life-threatening ventricular arrhythmias, especially if the accessory pathway has a short anterograde refractory period $(<250 \mathrm{~ms})$. Role of CA in such patients is wellestablished and carries a Class I recommendation from the ACC. Additionally, the ESC guidelines state that urgent CA is recommended both for primary and secondary prevention in patients in case of cardiac arrest as a Class 1 indication and may be considered in asymptomatic patients with overt pre-excitation after careful counseling (Class IIa).

4. AF in athletes: There exists a U-shaped relationship of AF occurrence with exercise, and people at both ends of the spectrum are at high risk of both paroxysmal and persistent AF. They tend to be mostly vagal mediated, with atrial dilatation and chronic inflammation potentially playing a role. Studies are divided on the benefit of CA in athletes with some suggesting definite benefit, while others have found CA to be comparable to drug therapy, although this might be challenging due to profound bradycardia in this population. CA currently carries a Class IIa recommendation as first-line therapy in athletes based on professional society guidelines.

5. AF in adults with congenital heart disease: Development of early and late tachyarrhythmias is fairly common in unrepaired atrial septal defects, patients with Fontan circulation and patients with repaired tetralogy of Fallot. Pathogenesis is complex but can be related to hypertrophy and fibrosis of the atria, volume overload state or from scar-related formation of macro-reentry circuits. In such patients, long-term use of AAD can be challenging due to side-effect profile. CA has been shown to be effective in small cohorts of such patients with symptomatic AF, but strong evidence is lacking. At the present time, CA has a Class IIb indication in symptomatic patients with congenital heart disease when performed in experienced centers.

\section{Conclusion}

CA is a relatively safe procedure and has proven benefit in alleviating symptoms and improving QOL in patients with symptomatic paroxysmal and persistent AF. CA might have mortality benefit in certain select populations such as younger patients and those with systolic HF. Success of procedure depends upon AF chronicity and comorbid conditions, and outcomes are generally better in experienced centers with high volumes and operator experience. Future studies targeting pathways for neurohormonal and autonomic modulation in addition to pulmonary vein isolation could help explore new avenues in the management of patients with symptomatic AF.

\section{List of Acronyms}

RAAFT Radiofrequency ablation vs antiarrhythmic drugs as first-line treatment of paroxysmal atrial fibrillation

CABANA Catheter Ablation versus Antiarrhythmic Drug Therapy for Atrial Fibrillation 
AATAC

Ablation vs. Amiodarone for Treatment of Atrial Fibrillation in Patients With Congestive Heart Failure and an Implanted ICD/CRTD study

PABA CHF Pulmonary-Vein Isolation for Atrial Fibrillation in Patients with Heart Failure study

CASTLE-AF Catheter Ablation for Atrial Fibrillation with Heart Failure

Conflict of Interest

None declared.

\section{References}

1 January CT, Wann LS, Alpert JS, et al; American College of Cardiology/American Heart Association Task Force on Practice Guidelines. 2014 AHA/ACC/HRS guideline for the management of patients with atrial fibrillation: a report of the American College of Cardiology/American Heart Association Task Force on Practice Guidelines and the Heart Rhythm Society. J Am Coll Cardiol 2014; 64(21):e1-e76

2 Kirchhof P, Benussi S, Kotecha D, et al. 2016 ESC Guidelines for the management of atrial fibrillation developed in collaboration with EACTS. Europace 2016;18(11):1609-1678

3 Calkins H, Hindricks G, Cappato R, et al. 2017 HRS/EHRA/ECAS/ APHRS/SOLAECE expert consensus statement on catheter and surgical ablation of atrial fibrillation: executive summary. J Arrhythm 2017;33(05):369-409

4 Wilber DJ, Pappone C, Neuzil P, et al; ThermoCool AF Trial Investigators. Comparison of antiarrhythmic drug therapy and radiofrequency catheter ablation in patients with paroxysmal atrial fibrillation: a randomized controlled trial. JAMA 2010;303 (04):333-340

5 Packer DL, Kowal RC, Wheelan KR, et al; STOP AF Cryoablation Investigators. Cryoballoon ablation of pulmonary veins for paroxysmal atrial fibrillation: first results of the North American Arctic Front (STOP AF) pivotal trial. J Am Coll Cardiol 2013;61(16):1713-1723

6 Pappone C, Augello G, Sala S, et al. A randomized trial of circumferential pulmonary vein ablation versus antiarrhythmic drug therapy in paroxysmal atrial fibrillation: the APAF Study. J Am Coll Cardiol 2006;48(11):2340-2347

7 Jaïs P, Cauchemez B, Macle L, et al. Catheter ablation versus antiarrhythmic drugs for atrial fibrillation: the A4 study. Circulation 2008;118(24):2498-2505

8 Di Biase L, Mohanty P, Mohanty S, et al. Ablation versus amiodarone for treatment of persistent atrial fibrillation in patients with congestive heart failure and an implanted device: results from the AATAC multicenter randomized trial. Circulation 2016;133(17): 1637-1644

9 Padfield GJ, Steinberg C, Swampillai J, et al. Progression of paroxysmal to persistent atrial fibrillation: 10-year follow-up in the Canadian Registry of Atrial Fibrillation. Heart Rhythm 2017;14(06):801-807

10 Wazni OM, Marrouche NF, Martin DO, et al. Radiofrequency ablation vs antiarrhythmic drugs as first-line treatment of symptomatic atrial fibrillation: a randomized trial. JAMA 2005;293 (21):2634-2640

11 Cosedis Nielsen J, Johannessen A, Raatikainen P, et al. Radiofrequency ablation as initial therapy in paroxysmal atrial fibrillation. N Engl J Med 2012;367(17):1587-1595

12 Morillo CA, Verma A, Connolly SJ, et al; RAAFT-2 Investigators. Radiofrequency ablation vs antiarrhythmic drugs as first-line treatment of paroxysmal atrial fibrillation (RAAFT-2): a randomized trial. JAMA 2014;311(07):692-700

13 Packer DL, Mark DB, Robb RA, et al; CABANA Investigators. Effect of catheter ablation vs antiarrhythmic drug therapy on mortality, stroke, bleeding, and cardiac arrest among patients with atrial fibrillation: the CABANA randomized clinical trial. JAMA 2019; 321(13):1261-1274

14 Noseworthy PA, Gersh BJ, Kent DM, et al. Atrial fibrillation ablation in practice: assessing CABANA generalizability. Eur Heart J 2019;40(16):1257-1264

15 Carlisle MA, Fudim M, DeVore AD, Piccini JP. Heart failure and atrial fibrillation, like fire and fury. JACC Heart Fail 2019;7(06): 447-456

16 Jones DG, Haldar SK, Hussain W, et al. A randomized trial to assess catheter ablation versus rate control in the management of persistent atrial fibrillation in heart failure. J Am Coll Cardiol 2013;61(18):1894-1903

17 Khan MN, Jaïs P, Cummings J, et al; PABA-CHF Investigators. Pulmonary-vein isolation for atrial fibrillation in patients with heart failure. N Engl J Med 2008;359(17):1778-1785

18 Hunter RJ, Berriman TJ, Diab I, et al. A randomized controlled trial of catheter ablation versus medical treatment of atrial fibrillation in heart failure (the CAMTAF trial). Circ Arrhythm Electrophysiol 2014;7(01):31-38

19 Marrouche NF, Brachmann J, Andresen D, et al; CASTLE-AF Investigators. Catheter ablation for atrial fibrillation with heart failure. N Engl J Med 2018;378(05):417-427

20 Prabhu S, Taylor AJ, Costello BT, et al. Catheter ablation versus medical rate control in atrial fibrillation and systolic dysfunction: the CAMERA-MRI study. J Am Coll Cardiol 2017;70(16):1949-1961

21 Douglas L. Packer, Hussein R. Al-Khalidi, Adam P. Silverstein, Jeanne E. Poole, Tristam D. Bahnson, Daniel B. Mark, Kerry L. Lee. Dealing with the double whammy: AF in HF. Heart Rhythm Society Annual Scientific Sessions; 2019; San Fransisco, CA

22 January CT, Wann LS, Calkins H, et al; Writing Group Members. 2019 AHA/ACC/HRS focused update of the 2014 AHA/ACC/HRS guideline for the management of patients with atrial fibrillation: a report of the American College of Cardiology/American Heart Association Task Force on Clinical Practice Guidelines and the Heart Rhythm Society. Heart Rhythm 2019;16(08):e66-e93

23 Black-Maier E, Ren X, Steinberg BA, et al. Catheter ablation of atrial fibrillation in patients with heart failure and preserved ejection fraction. Heart Rhythm 2018;15(05):651-657 
